\title{
Predisposição dos transtornos ansiosos em profissionais de saúde: uma revisão integrativa
}

Predisposition of anxiety disorders in health professionals: an integrative review

Predisposición de los trastornos de ansiedad en los profesionales de la salud: una revisión integradora

Recebido: 29/09/2021 | Revisado: 04/10/2021 | Aceito: 08/10/2021 | Publicado: 11/10/2021

Dayse de Araújo Silva Ferreira

ORCID: https://orcid.org/0000-00026269-5560 Centro Universitário de Ciências e Tecnologia do Maranhão, Brasil E-mail: dayse96.da@gmail.com

Karine Costa Melo

ORCID: https://orcid.org/0000-0001-8253-859X

Universidade Federal do Maranhão, Brasil E-mail: karinemelo09@gmail.com

Layse Siqueira Costa Miranda

ORCID: https://orcid.org/0000-0001-5942-4666 Centro Universitário de Ciências e Tecnologia do Maranhão, Brasil E-mail: layse62@gmail.com

Amanda Thais Franco Oliveira

ORCID: https://orcid.org/0000-0002-4210-7485 Centro Universitário de Ciências e Tecnologia do Maranhão, Brasil

E-mail: Amandafrancooliv@ hotmail.com

Linccon Fricks Hernandes

ORCID: https://orcid.org/0000-0002-7642-3080 Escola Superior de Ciências da Santa Casa de Misericórdia de Vitória, Brasil E-mail: fricksjr@hotmail.com

Dayanna Max Magalhães Bomfim ORCID: https://orcid.org/0000-0002-2883-4926 Faculdade Maurício de Nassau, Brasil E-mail: dayannamax@gmail.com

Eduardo Henrique Barros Ferreira ORCID: https://orcid.org/0000-0002-0072-3378

Universidad de San Lorenzo, Paraguai

E-mail: eh1405@hotmail.com

Kelly Rose Pinho Moraes

ORCID: https://orcid.org/0000-0002-5784-4939

Universidade Estadual do Maranhão, Brasil

E-mail: kelly.rose125@gmail.com

Ana Paula Cunha Duarte

ORCID: https://orcid.org/0000-0002-2898-5388

Universidade Federal do Maranhão, Brasil

E-mail: anapduarte002@gmail.com

Jairina Nunes Chaves

ORCID: https://orcid.org/0000-0002-3547-6901

Universidade Estadual do Maranhão, Brasil

E-mail: inanunes@hotmail.com

Caroline Natielle Rocha da Silva

ORCID: https://orcid.org/0000-0003-1323-7114

Universidade Federal do Maranhão, Brasil

E-mail: carollnathy@hormail.com

Paulo Sérgio Gaspar dos Santos

ORCID: https://orcid.org/0000-0003-1763-642X Centro Universitário de Ciências e Tecnologia do Maranhão, Brasil

E-mail: institutogasph@gmail.com

Ana Tereza Santos Dias de Almeida

ORCID: https://orcid.org/0000-0001-9927-1836 Centro Universitário de Ciências e Tecnologia do Maranhão, Brasil

E-mail: aterezadias@gmail.com

Patricia da Silva Pereira dos Reis

ORCID: https://orcid.org/0000-0002-4281-7540

Universidade Federal do Maranhão, Brasil

E-mail: patriciareissilvap@gmail.com 


\author{
Débora de Araújo Silva \\ ORCID: https://orcid.org/0000-0003-3472-6427 \\ Centro Universitário de Ciências e Tecnologia do Maranhão, Brasil \\ E-mail: arobed674@gmail.com \\ Surama Almeida Oliveira \\ ORCID: https://orcid.org/0000-0002-9746-5765 \\ Centro Universitário de Ciências e Tecnologia do Maranhão, Brasil \\ E-mail: su.a.oliveira@hotmail.com
}

\begin{abstract}
Resumo
Ansiedade é um sinal biológico que o organismo utiliza como proteção frente a uma ameaça. É uma emoção que é estimulada pela expectativa de um evento, real ou não, que possa ameaçar a conservação do organismo, onde a demonstração de ansiedade e estresse é considerada normal até o ponto de não provocar um sofrimento ao indivíduo, pois o sofrimento pode causar uma ansiedade patológica e resultar posteriormente nos chamados transtornos ansiosos, o objetivo da pesquisa foi identificar fatores que predispõem a ocorrência dos transtornos ansiosos em profissionais de saúde. O é uma pesquisa bibliográfica do tipo revisão integrativa da literatura. Quanto aos resultados, os profissionais da saúde são suscetíveis ao aparecimento de transtornos ansiosos e outros transtornos mentais, onde o fato se deve a associação de diversos fatores de risco como: idade, sexo, estado civil e presença de doenças crônicas e outros. Conclui-se que os profissionais da saúde também precisam receber assistência física e psicológica, além de apoio social e implementação de estratégias que visem minimizar o risco de aparecimento de transtornos mentais.
\end{abstract}

Palavras-chave: Transtorno de ansiedade; Pessoal da saúde; Covid-19; Transtornos mentais.

\begin{abstract}
Anxiety is a biological signal that the body uses to protect against a threat. It is an emotion that is stimulated by the expectation of an event, real or not, that may threaten the conservation of the organism, where the demonstration of anxiety and stress is considered normal to the point of not causing suffering to the individual, as suffering can cause a pathological anxiety and later result in the so-called anxiety disorders, the objective of the research was to identify factors that predispose the occurrence of anxiety disorders in health professionals. is a bibliographic research of the integrative literature review type. As for the results, health professionals are susceptible to the appearance of anxiety disorders and other mental disorders, where the fact is due to the association of several risk factors such as: age, gender, marital status and the presence of chronic diseases and others. It is concluded that health professionals also need to receive physical and psychological assistance, in addition to social support and implementation of strategies aimed at minimizing the risk of developing mental disorders.
\end{abstract}

Keywords: Anxiety disorders; Health personnel; Covid-19; Mental disorders.

\title{
Resumen
}

La ansiedad es una señal biológica que el cuerpo usa para protegerse contra una amenaza. Es una emoción que es estimulada por la expectativa de un evento, real o no, que pueda amenazar la conservación del organismo, donde la demostración de ansiedad y estrés se considera normal al punto de no causar sufrimiento al individuo, como sufrimiento. puede provocar una ansiedad patológica y posteriormente dar lugar a los denominados trastornos de ansiedad, el objetivo de la investigación fue identificar los factores que predisponen la ocurrencia de los trastornos de ansiedad en los profesionales de la salud. es una investigación bibliográfica del tipo revisión integradora de la literatura. En cuanto a los resultados, los profesionales de la salud son susceptibles a la aparición de trastornos de ansiedad y otros trastornos mentales, donde el hecho se debe a la asociación de varios factores de riesgo como: edad, sexo, estado civil y presencia de enfermedades crónicas y otros. Se concluye que los profesionales de la salud también necesitan recibir asistencia física y psicológica, además del apoyo social y la implementación de estrategias encaminadas a minimizar el riesgo de desarrollar trastornos mentales.

Palabras clave: Ansiedad de separación; Personal de salud; Covid-19; Trastornos mentales.

\section{Introdução}

Ansiedade é um sinal biológico que o organismo utiliza como proteção frente a uma ameaça. É uma emoção que é estimulada pela expectativa de um evento, real ou não, que possa ameaçar a conservação do organismo, onde a demonstração de ansiedade e estresse é considerada normal até o ponto de não provocar um sofrimento ao indivíduo, pois o sofrimento pode causar uma ansiedade patológica e resultar posteriormente nos chamados transtornos ansiosos (Santana \& Bião, 2018). Destaca-se que dentre os transtornos psiquiátricos, os transtornos ansiosos são mais predominantes, onde pesquisas mostram que o gênero feminino tem maior risco de desenvolver os transtornos ao longo da vida em comparação aos homens (Machado \& Quevedo 2016).

Os transtornos ansiosos cobrem vários aspectos da personalidade psicológica e emocional das pessoas, envolvendo as 
possibilidades diagnósticas de muitas outras doenças, neste aspecto, a perspectiva multidisciplinar torna-se extremamente importante principalmente para os profissionais que atuam na área de saúde mental, para uma compreensão do quadro clínico geral do paciente, e a busca por um tratamento específico e um acompanhamento eficaz (Moura, Lunard, Volpato, \& Nascimento, 2018).

Deve-se destacar ainda, a influência da pandemia de Covid-19 sobre o surgimento de depressão, ansiedade, estresse, insônia e o agravamento de diversos transtornos psicológicos, com ênfase ainda na síndrome de Burnout, onde pessoas de diversas idades, gêneros e classes profissionais foram afetadas (Melo et al., 2021; Da Silva et al., 2021).

Diante disso, o tema a ser estudado vem de um interesse em cooperar com conhecimento dos transtornos ansiosos em profissionais da saúde em busca de um diagnóstico e tratamento precoce. Nesse sentindo esse estudo é de suma importância para estimular o interesse de outras pessoas e a preocupação com esses profissionais que estão a todo tempo propícios a passar por situações de desequilíbrio emocional, sugestivos a desencadear transtornos ansiosos.

Essa pesquisa se desenvolveu baseando-se na seguinte questão: "De acordo com as evidências científicas qual seria a prevalência e o fatores de riscos associados aos transtornos ansiosos em profissionais de saúde?”. Assim, o objetivo geral desse estudo foi identificar fatores que predispõem a ocorrência dos transtornos ansiosos em profissionais de saúde. Já os objetivos específicos foram: avaliar a prevalência dos principais transtornos ansiosos em comparação a outros transtornos mentais; destacar estratégias de prevenção/proteção para transtornos ansiosos em profissionais da saúde; e relacionar a pandemia de Covid-19 com o aumento de transtornos ansiosos nos profissionais da saúde.

\section{Metodologia}

O presente estudo tratou-se de uma pesquisa bibliográfica do tipo revisão integrativa da literatura, na qual utilizou-se a metodologia proposta por Alves e Oliveira (2016). Este procedimento foi escolhido por possibilitar a síntese e análise do conhecimento científico já produzido sobre o tema "PREDISPOSIÇÃO DOS TRANSTORNOS ANSIOSOS EM PROFISSIONAIS DE SAÚDE: uma revisão integrativa".

A revisão da literatura abrangente inclui uma análise extensa da literatura, o que ajuda a discutir métodos e resultados de pesquisas e refletir sobre pesquisas futuras. O objetivo original deste método de pesquisa foi aprofundar a compreensão de um determinado assunto com base em pesquisas anteriores. Este método fornece uma combinação de dados da literatura teórica e empírica, para se ter um melhor entendimento do tema de interesse (Sousa, Vieira, Severino, \& Antunes, 2017).

O tema "PREDISPOSIÇÃO DOS TRANSTORNOS ANSIOSOS EM PROFISSIONAIS DE SAÚDE: uma revisão integrativa", estabeleceu a construção da estratégia PICO, que representa um acrônimo para Paciente (P), Intervenção (I), Comparação (C) e Desfechos (O-outcomes), na qual foi utilizada para a geração da questão norteadora desta revisão integrativa da literatura: "De acordo com as evidências cientificas qual seria a prevalência e o fatores de riscos associados aos transtornos ansiosos em profissionais de saúde?".

Para a realização da coleta de dados bibliográficos foi utilizado um recorte temporal de 2016 a 2020, utilizando os descritores tais como os DECS (Descritores em Ciências da Saúde) que disponibiliza um vocabulário estruturado nos idiomas inglês, espanhol e português, o MESH terms (Medical Subject Headings), e os títulos CINHAL (Cumulative Index to Nursing and Allied Health Literature) conforme o Quadro 1. 
Quadro 1. Elementos da estratégia PICO, descritores e palavras-chave utilizados - Caxias, MA, Brasil, 2021.

\begin{tabular}{|l|c|c|c|c|c|}
\hline \multicolumn{2}{|c|}{ ELEMENTOS } & DECS & MESH & CINAHL & $\begin{array}{c}\text { PALAVRAS- } \\
\text { CHAVES }\end{array}$ \\
\hline P & Profissionais da & Pessoal de Saúde & Health & Health Personnel & - \\
& saúde & Health Personnel & & \\
& & Personal de Salud & & \\
\hline I & Prevalência & Prevalência & Prevalence & Prevalence & Risk Factors \\
& Fatores de risco & Prevalence Prevalencia & Risk Factors & & \\
& & Fatores de Risco & & \\
& & Risk Factors & & \\
& & Factores de Riesgo & & & \\
\hline Co & Transtornos ansiosos & Transtornos de Ansiedade & Anxiety & Anxiety Disorders & \\
& & Anxiety Disorders & Disorders & & \\
& & Trastornos de Ansiedad & & \\
\hline
\end{tabular}

Fonte: Descritores e Palavras-chaves (2021).

A partir dos descritores definidos, estes foram aplicados nas bases de dados e combinados, não foram utilizadas palavras-chave, a combinação dos termos resultou em estratégia de busca que foram especificas em cada bases utilizadas, como pode ser observado no Quadro 2.

Quadro 2. Estratégias de busca utilizadas nas bases de dados BIREME, PUBMED e CINAHL - Caxias, MA, Brasil, 2021.

\begin{tabular}{|c|c|c|c|c|}
\hline $\begin{array}{c}\text { BASE DE } \\
\text { DADOS }\end{array}$ & ESTRATÉGIA DE BUSCA & 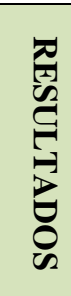 & 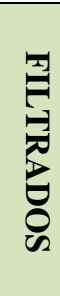 & 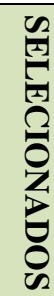 \\
\hline $\begin{array}{l}\text { Bireme } \\
\text { (Decs) }\end{array}$ & $\begin{array}{l}\text { (Health Personnel) AND (prevalence) AND (risk factors) AND (Anxiety } \\
\text { Disorders) }\end{array}$ & 272 & 75 & 7 \\
\hline $\begin{array}{c}\text { PubMed } \\
\text { (Mesh terms) }\end{array}$ & $\begin{array}{l}\text { (((Health Personnel) AND (prevalence)) AND (risk factors)) AND (Anxiety } \\
\text { Disorders) }\end{array}$ & 395 & 95 & 04 \\
\hline $\begin{array}{c}\text { Cinahl } \\
\text { (Título Cinahl) }\end{array}$ & health personnel AND prevalence AND risk factors AND anxiety disorders & 12 & 02 & 01 \\
\hline
\end{tabular}

Fonte: Bases de dados (2021).

Como critérios de inclusão, foram utilizados estudos disponíveis em sua totalidade, publicados entre os anos de 2016 a 2020, nos idiomas Português, Inglês e Espanhol, e que atendessem a questão norteadora proposta neste estudo. Foram excluídos da busca inicial capítulos de livros, resumos, textos incompletos, teses de doutorado, dissertações de mestrados, monografias, relatos técnicos e outras formas de publicação que não se adequam a artigos científicos completos.

A análise dos estudos foi dividida em duas fases: na primeira, os estudos foram pré-selecionados segundo os critérios de inclusão e exclusão e de acordo com a estratégia formulada e o endereço de busca correspondente de cada base de dados.

Foram encontrados duzentos e setenta e dois (272) estudos como busca geral na BVS, sendo que limitando a busca para artigos com texto completo, recorte temporal dos últimos cinco anos, e assunto principal (Pessoal de Saúde, Ansiedade Transtornos de Estresse Pós-Traumáticos, Transtornos de Ansiedade) obtiveram-se setenta e cinco (75) estudos, destes foram analisados títulos e resumos sendo selecionados quarenta e dois (42) estudos, após a leitura na íntegra apenas sete (07) estudos foram condizentes com a questão desta pesquisa.

Na base PUBMED, como busca total foram encontrados trezentos e noventa e cinco (395) estudos, aplicando na pesquisa o filtro que limita por texto completo dos últimos cinco anos, obtiveram-se noventa e cinco (95) estudos, destes foram 
analisados títulos e resumos e selecionados vinte e um (21) estudo, após a leitura dos textos obteve como resultado final quatro (04) estudos.

Foram encontrados na Cinahl doze (12) estudos no total, e após aplicação dos filtros resultaram em dois (02) estudos, após a leitura dos títulos e resumos somente um (01) estudo foi incluído. Ao final da análise treze (12) artigos foram selecionados na amostra final desta revisão integrativa.

Posteriormente, foram analisadas as informações coletadas nos artigos científicos e criadas categorias analíticas que facilitaram a ordenação e a sumarização de cada estudo. Essa categorização foi realizada de forma descritiva, indicando os dados mais relevantes para o estudo. A pesquisa levou em consideração os aspectos éticos quanto às citações dos estudos, respeitando a autoria das ideias, os conceitos e as definições presentes nos artigos incluídos na revisão.

Optou-se pela análise em forma estatística e de forma de texto, utilizando cálculos matemáticos e inferências, que foram apresentados em quadros e tabelas para facilitar a visualização e compreensão. As evidências científicas foram classificadas segundo os níveis e graus de recomendação propostos por Bork (2011).

\section{Resultados}

Essa fase é organizada em duas partes. A primeira está relacionada com a caracterização dos estudos, já a segunda, relaciona-se ao cumprimento do objetivo do estudo, que consiste em conhecer os transtornos de ansiedade em profissionais da saúde.

\section{Caracterização dos estudos}

Dos 12 artigos incluídos nesta revisão, todos apresentaram abordagem quantitativa dos dados (100\%); em que oito (67\%) concentram-se principalmente no ano de 2020; e todos os estudos estavam na língua inglesa (100\%). Sobre a procedência houve predomínio de estudos realizados na China, correspondente a três $(25 \%)$ trabalhos. No tocante ao periódico, o Plos One apresentou maior número de publicações, correspondendo a 17\%, os demais periódicos obtiveram 8\% cada. Em relação ao delineamento de pesquisa e nível e evidência, houve prevalência de estudos transversais (92\%), representando nível de evidencia seis; a maior parte dos estudos (75\%) obtiveram grau de recomendação A para a prática clínica, conforme demonstrado na Tabela 1.

Tabela 1. Análise descritiva das produções científicas acerca dos transtornos de ansiedade em profissionais da saúde. Caxias, MA, Brasil, 2021. $(\mathrm{N}=12)$

\begin{tabular}{|c|c|c|}
\hline Variáveis & $\mathbf{N}$ & $\%$ \\
\hline \multicolumn{3}{|l|}{ Base de dados } \\
\hline BIREME & 07 & 58,0 \\
\hline PUBMED & 04 & 33,0 \\
\hline CINAHL & 01 & 8,0 \\
\hline \multicolumn{3}{|l|}{ Abordagem do estudo } \\
\hline Quantitativo & 12 & 100,0 \\
\hline \multicolumn{3}{|l|}{ Ano } \\
\hline 2016 & 01 & 8,0 \\
\hline 2018 & 01 & 8,0 \\
\hline 2020 & 08 & 67,0 \\
\hline 2021 & 02 & 17,0 \\
\hline \multicolumn{3}{|l|}{ Idioma } \\
\hline Inglês & 12 & 100,0 \\
\hline \multicolumn{3}{|l|}{ País } \\
\hline África do Sul & 01 & 8,0 \\
\hline China & 03 & 25,0 \\
\hline
\end{tabular}




\begin{tabular}{lcc}
\hline Coreia & & 8,0 \\
Espanha & 01 & 8,0 \\
Grécia & 01 & 8,0 \\
Paquistão & 01 & 8,0 \\
Turquia & 01 & 17,0 \\
Etiópia & 02 & 8,0 \\
França & 01 & 8,0 \\
Periódicos & 01 & 17,0 \\
Plos One & 02 & 8,0 \\
Journal of Affective Disorders & 01 & 8,0 \\
Translational Psychiatry & 01 & 8,0 \\
Preventive \& Social Medicine & 01 & 8,0 \\
Archives of Environmental \& Occupational Health & 01 & 8,0 \\
Medical Archives & 01 & 8,0 \\
BMC Psychiatry & 01 & 8,0 \\
Journal of Affective Disorders & 01 & 8,0 \\
Journal of Community Health & 01 & 8,0 \\
Journal of Psychiatric Research & 01 & 8,0 \\
American Journal of Respiratory and Critical Care Medicine & 01 & 8,0 \\
Delineamento de pesquisa & & 92,0 \\
Caso Controle & 01 & 8,0 \\
Estudo transversal & 11 & 92,0 \\
Classificação da evidência & & 75,0 \\
Nível 04 Nível 06 & 01 & 25,0 \\
Grau de recomendação & 11 & \\
A & & \\
B
\end{tabular}

Legenda: N = número; \% = percentual. Fonte: Dados da pesquisa (2021).

O Quadro 3 mostra a distribuição dos estudos segundo autores e anos de publicação, o autor; título; tipo de estudo; objetivo e perfil amostral. A maioria dos estudos versaram sobre os fatores associados aos efeitos psicológicos em profissionais de saúde, principalmente durante a pandemia de Covid-19, em que os transtornos de ansiedade encontrados foram: transtorno de ansiedade generalizada; e transtorno do estresse pós traumático.

Além disso outros distúrbios psicológicos foram analisados, como: depressão; transtorno por uso de álcool; estresse emocional; desesperança; distúrbios psicológicos de somatização; insônia; e risco de suicídio. Foi analisado os fatores de risco, fatores relacionados (que afetam os níveis de ansiedade), prevalência, incidência e a percepção desses profissionais acerca desses distúrbios. 
Quadro 3. Publicações incluídas segundo o título do artigo, autor, objetivo principal e perfil amostral. Caxias, MA, Brasil. 2021. (N=12)

\begin{tabular}{|c|c|c|c|c|}
\hline $\begin{array}{l}N^{\circ} \text { DE } \\
\text { ORDEM } \\
\text { E BASE }\end{array}$ & TÍTULO DO ARTIGO & AUTOR/ ANO & OBJETIVO PRINCIPAL & PERFIL AMOSTRAL \\
\hline $\begin{array}{l}\text { A1 } \\
\text { BIREME }\end{array}$ & $\begin{array}{l}\text { Socio-ecological predictors of } \\
\text { mental health outcomes among } \\
\text { healthcare workers during the } \\
\text { Covid-19 pandemic in the United } \\
\text { States. }\end{array}$ & $\begin{array}{l}\text { Hennein, Mew e } \\
\text { Lowe (2021) }\end{array}$ & $\begin{array}{l}\text { Avaliar fatores socioecológicos associados } \\
\text { a quatro resultados de saúde mental: } \\
\text { depressão maior; transtorno de ansiedade } \\
\text { generalizada; transtorno de estresse pós- } \\
\text { traumático; e transtorno por uso de álcool } \\
\text { em profissionais de saúde durante a } \\
\text { pandemia Covid-19. }\end{array}$ & $\begin{array}{l}\text { Estudo realizado com médicos, estagiários de medicina, enfermeiros, assistentes } \\
\text { clínicos, técnicos / técnicos de saúde e pessoal não clínico. Um total de } 1132 \\
\text { profissionais de saúde participaram; destes, } 1.092(96,5 \%) \text { completaram todas as } \\
\text { medidas do atual estudo e constituíram a amostra analítica. }\end{array}$ \\
\hline $\begin{array}{l}\text { A2 } \\
\text { BIREME }\end{array}$ & $\begin{array}{l}\text { Anxiety and depression symptoms } \\
\text { of medical staff under Covid-19 } \\
\text { epidemic in China. }\end{array}$ & Liu et al. (2021) & $\begin{array}{l}\text { Investigar anonimamente os sintomas de } \\
\text { ansiedade e depressão entre a equipe } \\
\text { médica durante o surto de Covid-19. }\end{array}$ & $\begin{array}{l}\text { Um total de } 1.090 \text { equipes médicas foram investigadas neste estudo. Sendo } 216 \\
\text { homens e } 874 \text { mulheres. }\end{array}$ \\
\hline $\begin{array}{c}\text { A3 } \\
\text { BIREME }\end{array}$ & $\begin{array}{l}\text { Acute psychological effects of } \\
\text { Coronavirus Disease } 2019 \\
\text { outbreak among healthcare } \\
\text { workers in China: a cross-sectional } \\
\text { study }\end{array}$ & $\begin{array}{l}\text { Wang, Ma, } \\
\text { Yang, \& Cai. } \\
\text { (2020) }\end{array}$ & $\begin{array}{l}\text { Avaliar os efeitos psicológicos agudos } \\
\text { experimentados por profissionais de saúde } \\
\text { durante o período inicial do surto de Covid- } \\
19 \text { na China }\end{array}$ & $\begin{array}{l}897 \text { profissionais de saúde consistiam em } 332 \text { homens }(17,5 \%) \text { e } 1565 \text { mulheres } \\
(82,5 \%) \text {. A maioria dos PS tinha entre } 25 \text { e } 40 \text { anos. Quase metade dos profissionais de } \\
\text { saúde }(49,5 \%) \text { foram recrutados em hospitais em Wuhan, } 29,7 \% \text { eram médicos, } 70,3 \% \\
\text { eram enfermeiras, } 76,3 \% \text { trabalhavam em hospitais terciários e } 39,1 \% \text { trabalhavam na } \\
\text { linha de frente contra Covid- } 19 \text {. }\end{array}$ \\
\hline $\begin{array}{c}\text { A4 } \\
\text { BIREME }\end{array}$ & $\begin{array}{lccr}\text { Covid-19 } & \text { Outbreak } & \text { and Its } \\
\text { Association } & \text { with } & \text { Healthcare } \\
\text { Workers' Emotional } & \text { Stress: a } \\
\text { Cross-Sectional Study } & \end{array}$ & Park et al. (2020) & $\begin{array}{l}\text { Avaliar os correlatos imediatos de estresse } \\
\text { emocional e identificar quais trabalhos, } \\
\text { departamentos e tipos de exposição } \\
\text { específicos são fatores de risco para } \\
\text { estresse emocional em profissionais de } \\
\text { saúde. }\end{array}$ & $\begin{array}{l}\text { Foram analisados os dados de } 1.003 \text { participantes. Dos participantes, } 352(35,1 \%) \\
\text { tinham } 20 \text { anos, } 776(77,4 \%) \text { tinham entre } 20 \text { e } 50 \text { anos e a maioria }(\mathrm{n}=773 ; 77,1 \%) \\
\text { eram mulheres. Além disso, } 710(70,8 \%) \text { possuíam o título de bacharel e a maioria }(\mathrm{n}= \\
648 ; 64,6 \%) \text { eram enfermeiras. }\end{array}$ \\
\hline $\begin{array}{l}\text { A5 } \\
\text { BIREME }\end{array}$ & $\begin{array}{l}\text { Anxiety and depression predict } \\
\text { musculoskeletal disorders in } \\
\text { healthcare workers }\end{array}$ & $\begin{array}{l}\text { Tsaras et al. } \\
\text { (2016) }\end{array}$ & $\begin{array}{l}\text { Determinar se a incidência de distúrbios } \\
\text { musculoesqueléticos estão associados a } \\
\text { ansiedade e / ou depressão preexistentes em } \\
\text { profissionais de saúde do sexo feminino. }\end{array}$ & $\begin{array}{l}\text { Este estudo foi realizado no Hospital Universitário Fundación Jiménez Díaz, de } \\
\text { atendimento terciário. A população do estudo foi composta por trabalhadores da área } \\
\text { de saúde, incluindo médicas, enfermeiras, técnicas (laboratório, radiologia e } \\
\text { fisioterapia) e auxiliares de enfermagem. Sendo composto por profissionais do sexo } \\
\text { feminino, sendo } 56 \text { casos / } 55 \text { controles. }\end{array}$ \\
\hline $\begin{array}{c}\text { A11 } \\
\text { PUBMED }\end{array}$ & $\begin{array}{l}\text { Symptoms of Anxiety, Depression, } \\
\text { and Peritraumatic Dissociation in } \\
\text { Critical Care Clinicians Managing } \\
\text { Patients with Covid-19 A Cross- } \\
\text { Sectional Study }\end{array}$ & $\begin{array}{l}\text { Azoulay et al. } \\
\quad(2020)\end{array}$ & 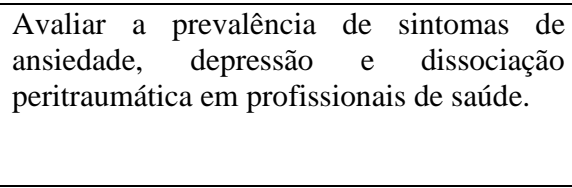 & $\begin{array}{l}\text { Entre os } 1.580 \text { profissionais de saúde que trabalham nas } 21 \text { UTI, } 1.058(67 \%) \\
\text { responderam totalmente a pesquisa. A idade média dos entrevistados foi } 33 \text { (IQR, 28- } \\
\text { 41) anos e } 71 \% \text { eram mulheres. Entre os entrevistados, } 721(68,3 \%) \text { faziam parte da } \\
\text { equipe de enfermagem, } 29,1 \% \text { eram médicos e } 2,6 \% \text { foram outros profissionais } \\
\text { aliados. }\end{array}$ \\
\hline $\begin{array}{c}\text { A12 } \\
\text { CINAHL }\end{array}$ & $\begin{array}{l}\text { The prevalence and risk factors of } \\
\text { psychological disturbances of } \\
\text { frontline medical staff in china } \\
\text { under the Covid-19 epidemic: } \\
\text { Workload should be concerned }\end{array}$ & $\begin{array}{l}\text { Zhou et al. } \\
\text { (2020) }\end{array}$ & $\begin{array}{l}\text { Investigar a prevalência e os fatores de } \\
\text { risco (incluindo sobrecarga de trabalho) de } \\
\text { distúrbios psicológicos entre equipes } \\
\text { médicas de primeira linha na China, } \\
\text { incluindo depressão, ansiedade e sintomas } \\
\text { de somatização, insônia e risco de suicídio. }\end{array}$ & $\begin{array}{l}\text { Um total de } 606 \text { funcionários de hospitais de primeira linha e } 1.099 \text { pessoas comuns } \\
\text { foram recrutados em } 133 \text { cidades na China. } 450 \text { eram do sexo masculino e } 1255 \text { do } \\
\text { sexo feminino. Sendo que } 636 \text { faziam parte da equipe médica da linha de frente da } \\
\text { Covid-19. }\end{array}$ \\
\hline
\end{tabular}

Fonte: Bases de Dados (2021). 


\section{Caracterização das publicações quanto aos participantes da pesquisa e agravos encontrados}

Quanto ao perfil dos profissionais analisados, dos 12 estudos, $11(92 \%)$ especificaram a idade dos participantes, que variou entre 18 e >40 anos (Hennein, Mew, e Lowe, 2021; Liu et al., 2021; Wang et al., 2020; Park et al., 2020; Campo et al., 2016; Tsaras et al., 2018; Amin et al., 2020; Azoulay et al., 2020; Kibret et al., 2020; Hacimusalar et al., 2020; Sahin et al., 2020). Com relação ao sexo, dos 11 (92\%) estudos que pesquisaram com ambos os gêneros, houve predominância do sexo feminino (7.505/ 68\%) (Hennein, Mew, e Lowe, 2021; Liu et al., 2021; Wang et al., 2020; Park et al., 2020; Tsaras et al., 2018; Amin et al., 2020; Azoulay et al., 2020; Kibret et al., 2020; Hacimusalar et al., 2020; Sahin et al., 2020; Zhou et al., 2020) (Quadro 4).

No que concerne ao estado civil, este foi abordada em sete $(58 \%)$ estudos, sendo maior o número de profissionais casados (3.824/ 63\%) (Hennein, Mew, e Lowe, 2021; Liu et al., 2021; Wang et al., 2020; Park et al., 2020; Tsaras et al., 2018; Kibret et al., 2020; Zhou et al., 2020). A classe profissional foi relatada em 10 estudos, no qual houve predomínio de médicos (90\%) e enfermeiros (80\%) (Hennein, Mew, e Lowe, 2021; Liu et al., 2021; Wang et al., 2020; Park et al., 2020; Campo et al., 2016; Tsaras et al., 2018; Amin et al., 2020; Azoulay et al., 2020; Sahin et al., 2020; Zhou et al., 2020) (Quadro 4).

Os anos de experiência profissional foram incluídos em 6 (50\%) estudos, variando de menos de 5 anos a mais de 25 anos (Liu et al., 2021; Park et al., 2020; Campo et al., 2016; Tsaras et al., 2018; Kibret et al., 2020; Sahin et al., 2020) (Quadro 4).

Dos 10 artigos que foram analisados quanto aos transtornos ansiosos em profissionais da saúde, nove (90\%) apresentaram ansiedade ou sintomas de transtorno de ansiedade (Liu et al., 2021; Wang et al., 2020; Tsaras et al., 2018; Amin et al., 2018; Azoulay et al., 2020; Kibret et al., 2020; Hacimusalar et al., 2020; Sahin et al., 2020; Zhou et al., 2020). Um (10\%) estudo indicou estresse pós-traumático (Hennein, Mew, e Lowe, 2021). E duas (20\%) pesquisas apresentaram resultados com ansiedade generalizada (Wang et al., 2020; Hennein, Mew, e Lowe, 2021) (Quadro 5).

Com relação aos fatores de risco, três pesquisas (30\%) descreveram que ser do sexo feminino é um importante fator condicionante (Hennein, Mew, \& Lowe, 2021; Wang et al., 2020; Sahin et al., 2020). Dois estudos (20\%) apontaram a enfermagem (Liu et al., 2021; Wang et al., 2020), um (10\%) artigo indicou a presença de doença mental preexistente (Hennein, Mew, \& Lowe, 2021), e um (10\%) apontou o recebimento de treinamento inadequado (Wang et al., 2020) como importantes fatores de risco. Destacou-se ainda, fatores como: ser solteiro (Tsaras et al., 2018), um (10\%) artigo; ser casado (Liu et al., 2021), um (10\%) estudo; e ter pouco recurso para proteção (Wang et al., 2020), uma (10\%) pesquisa (Quadro 5).

Dentre os fatores de risco encontrados deve-se citar ainda que, dois (20\%) artigos mencionaram idade elevada (Liu et al., 2021; Tsaras et al., 2018); um (10\%) artigo relacionou ter filhos aos fatores condicionantes (Amin et al., 2018); Uma (10\%) pesquisa relatou a falta de experiência profissional (Tsaras et al., 2018) e ser muito jovem, um (10\%) estudo, (Amin et al., 2018) como possíveis fatores também (Quadro 5).

Um (10\%) não especificou os fatores (Hacimusalar et al., 2020), dois (20\%) estudos falaram da influência da renda familiar (Kibret et al., 2020; Zhou et al., 2020); um (10\%) artigo fala das doenças crônicas (Kibret et al., 2020); uma (10\%) pesquisa fala de doenças físicas (Zhou et al., 2020); e por fim, um (10\%) estudo fala da incapacidade de cuidar da família, arrependimentos e lutas contra emoções como fatores que oferecem risco (Azoulay et al., 2020) (Quadro 5). 
Research, Society and Development, v. 10, n. 13, e256101321238, 2021

(CC BY 4.0) | ISSN 2525-3409 | DOI: http://dx.doi.org/10.33448/rsd-v10i13.21238

Quadro 4. Caracterização dos estudos quanto ao perfil dos profissionais analisados. Caxias, MA, 2021.

\begin{tabular}{|c|c|c|c|c|c|c|c|c|}
\hline \multirow[t]{2}{*}{ Autor } & \multirow[t]{2}{*}{ Idade } & \multirow[t]{2}{*}{$\operatorname{Sexo}(\mathbf{M})$} & \multirow[t]{2}{*}{$\operatorname{Sexo}(\mathbf{F})$} & \multicolumn{3}{|c|}{ Estado civil } & \multirow[t]{2}{*}{ Classe profissional } & \multirow{2}{*}{$\begin{array}{c}\text { Anos de experiência } \\
\text { profissional }\end{array}$} \\
\hline & & & & Solteiro & Casado & $\begin{array}{c}\text { Divorciado } \\
\text { ou Viúvo }\end{array}$ & & \\
\hline $\begin{array}{l}\text { Hennein, Mew } \\
\text { e Lowe (2021) }\end{array}$ & Média $=40,44$ anos & 305 & 785 & 310 & 705 & 75 & $\begin{array}{l}\text { Médicos, enfermeiros, técnicos, estagiários e } \\
\text { outros. }\end{array}$ & Não reportado \\
\hline $\begin{array}{l}\text { Liu et al. } \\
\text { (2021) }\end{array}$ & $<30->40$ anos & 216 & 874 & 261 & 829 & 0 & Médicos, enfermeiros. & $<5->15$ anos \\
\hline $\begin{array}{l}\text { Wang et al. } \\
(2020)\end{array}$ & $18->40$ anos & 332 & 1565 & 617 & 1280 & 0 & Médicos, enfermeiros. & Não reportado \\
\hline $\begin{array}{c}\text { Park et al. } \\
(2020)\end{array}$ & $20-60$ anos & 230 & 773 & 494 & 491 & 0 & $\begin{array}{l}\text { Médicos, enfermeiros, técnicos, oficiais } \\
\text { administrativos, equipe da farmácia, outros. }\end{array}$ & $<5->25$ anos \\
\hline $\begin{array}{l}\text { Campo et al. } \\
\text { (2016) }\end{array}$ & Média $=42,5$ anos & - & 111 & \multicolumn{3}{|c|}{ Não reportado } & $\begin{array}{l}\text { Médicos, enfermeiros, técnicos e auxiliares } \\
\text { de enfermagem. }\end{array}$ & $7,1 \pm 7,6$ anos \\
\hline $\begin{array}{l}\text { Tsaras et al. } \\
\text { (2018) }\end{array}$ & $\begin{array}{l}\text { Média }=42,64 \pm \\
5,87\end{array}$ & 39 & 71 & 30 & 65 & 15 & $\begin{array}{l}\text { Enfermeiros, auxiliar de enfermagem e } \\
\text { chefes de departamento. }\end{array}$ & $15,73 \pm 5,64$ \\
\hline $\begin{array}{c}\text { Amin et al. } \\
(2020)\end{array}$ & Media $=35$ anos & 201 & 188 & \multicolumn{3}{|c|}{ Não reportado } & Médicos. & Não reportado \\
\hline $\begin{array}{l}\text { Azoulay et al. } \\
\qquad(2020)\end{array}$ & $28-41$ & 827 & 753 & \multicolumn{3}{|c|}{ Não reportado } & $\begin{array}{l}\text { Médicos, residentes, estudantes de medicina, } \\
\text { enfermeiros, auxiliar de enfermagem, } \\
\text { fisioterapeutas e psicólogos. }\end{array}$ & Não reportado \\
\hline $\begin{array}{l}\text { Kibret et al. } \\
(2020)\end{array}$ & $20-41$ & 104 & 201 & 290 & 0 & 0 & Não especificados. & $1-10$ anos \\
\hline $\begin{array}{l}\text { Hacimusalar } \\
\text { et al. (2020) }\end{array}$ & $18-70$ & 609 & 1547 & \multicolumn{3}{|c|}{ Não reportado } & Não especificado. & Não reportado \\
\hline $\begin{array}{l}\text { Sahin et al. } \\
(2020)\end{array}$ & $18-40$ & 620 & 319 & \multicolumn{3}{|c|}{ Não reportado } & Médicos, enfermeiros, outros. & \pm 10 anos \\
\hline $\begin{array}{l}\text { Zhou et al. } \\
\text { (2020) }\end{array}$ & Não reportado & 114 & 429 & 125 & 454 & 27 & Equipe médica da linha de frente. & Não reportado \\
\hline
\end{tabular}

Legenda: $\mathrm{M}=$ Masculino; $\mathrm{F}=$ Feminino. Fonte: Estudos analisados (2021). 
Quadro 5. Caracterização dos estudos quanto ao percentual de transtornos ansiosos e seus fatores de risco. Caxias, MA, 2021.

\begin{tabular}{|c|c|c|}
\hline Autor & Percentual de transtornos ansiosos & Fatores de risco \\
\hline $\begin{array}{l}\text { Hennein, Mew } \\
\text { e Lowe (2021) }\end{array}$ & $\begin{array}{l}15,6 \% \text { dos profissionais apresentaram provável transtorno de ansiedade } \\
\text { generalizada e } 22,8 \% \text { tinham provável transtorno de estresse pós- } \\
\text { traumático. }\end{array}$ & Ser do sexo feminino; ter doença mental pré-existente. \\
\hline $\begin{array}{l}\text { Liu et al. } \\
\text { (2021) }\end{array}$ & Houve prevalência de ansiedade em $13,3 \%$ dos participantes. & Ser casado; ter $<30$ anos; ser enfermeiro. \\
\hline $\begin{array}{l}\text { Wang et al. } \\
\quad(2020)\end{array}$ & $\begin{array}{l}\text { Houve prevalência de } 27,1 \% \text { de ansiedade e de } 9,8 \% \text { de Transtorno de } \\
\text { Ansiedade Generalizada. }\end{array}$ & $\begin{array}{l}\text { Ser do sexo feminino; ser enfermeiro, receber treinamento insuficiente e poucos recursos } \\
\text { para proteção. }\end{array}$ \\
\hline $\begin{array}{l}\text { Tsaras et al. } \\
\text { (2018) }\end{array}$ & Os profissionais apresentaram uma prevalência de $48,2 \%$ para ansiedade. & Ser solteiro; idade mais elevada; ter pouca experiência profissional. \\
\hline $\begin{array}{l}\text { Amin et al. } \\
(2020)\end{array}$ & Foi relatada uma prevalência de $43 \%$ de ansiedade. & Ser mais jovem; ter filhos. \\
\hline $\begin{array}{l}\text { Azoulay et al. } \\
\quad(2020)\end{array}$ & $9,1 \%$ dos participantes apresentaram sintomas de ansiedade. & Incapacidade de cuidar da própria família; arrependimentos; lutas com emoções. \\
\hline $\begin{array}{l}\text { Kibret et al. } \\
\quad(2020)\end{array}$ & A prevalência de ansiedade foi de $63 \%$. & Doenças crônicas; baixa renda familiar. \\
\hline $\begin{array}{l}\text { Hacimusalar et } \\
\text { al. }(2020)\end{array}$ & A prevalência de ansiedade foi de $91,5 \%$. & Não especificado. \\
\hline $\begin{array}{l}\text { Sahin et al. } \\
\quad(2020)\end{array}$ & Houve prevalência de ansiedade em $60,2 \%$ dos participantes. & Ser do sexo feminino. \\
\hline $\begin{array}{l}\text { Zhou et al. } \\
\text { (2020) }\end{array}$ & Foi relatado $45,4 \%$ de sintomas de ansiedade nos profissionais. & Renda familiar; doenças físicas. \\
\hline
\end{tabular}

Fonte: Estudos analisados (2021) 


\section{Discussão}

Fatores que predispõem a ocorrência dos transtornos ansiosos em profissionais de saúde e a relação da pandemia de covid-19 com o aumento dessa ocorrência

No estudo de Hennein, Mew e Lowe (2021), realizado com 1092 trabalhadores da saúde nos Estados Unidos no contexto da pandemia por Covid-19, evidenciaram os fatores prováveis relacionados ao Transtorno de Ansiedade Generalizada (TAG) e Transtorno do Estresse Pós-traumático (TEPT). Entre os associados para TAG e TEPT, encontraram-se: doença mental pré-pandêmica; aumento nas necessidades de suporte social; e aumento no uso diário de mídias sociais. Outros fatores associados a TAG: idade (para cada ano 0,97 chances); ser afro-americanos; e ser tecnólogo ou técnico em saúde. Para TEPT: ser do gênero feminino e percepção da estigmatização do profissional de saúde.

Com relação ao fator idade, Tsaras et al. (2018), obtiveram resultados semelhantes, no estudo realizado com 110 enfermeiras de saúde mental na Grécia, evidenciaram que a idade das enfermeiras foi um preditor positivo de transtorno de ansiedade. Especificamente, o risco de desenvolvimento de ansiedade aumentou $11 \%$ para cada ano de idade crescente. Os estudos Sahin et al. (2020) realizado na Turquia e o de Kibret et al. (2020) na Etiópia, obtiveram resultados semelhantes.

Os estudos mostram que o sexo feminino é mais suscetível aos transtornos ansiosos, como o estudo de Wang et al. (2020) realizado com 897 profissionais de saúde na China evidenciaram que os transtornos de ansiedade foram prevalentes em mulheres. Corroborando com os estudos de Sahin et al. (2020) e Hacimusalar et al. (2020). Contudo, observou-se que o maior público analisado por esses estudos eram profissionais do sexo feminino, o que pode ser um viés favorável para tal resultado.

Foi observada a presença de transtornos ansiosos em indivíduos com doença mental anterior no estudo de Hennein et al. (2021), esse dado também foi encontrado em um estudo realizado com 939 profissionais de saúde na Turquia em que todas as pontuações foram significativamente mais altas entre os participantes com histórico de doença psiquiátrica e que necessitavam de apoio psiquiátrico durante a pandemia de Covid-19 (Sahin et al., 2020).

Outros fatores foram observados por Liu et al. (2021), em um estudo realizado com um total de 1.090 profissionais na China, observaram que proporções significativamente maiores associadas aos sintomas de ansiedade autorrelatados incluíam o estado de casado, nunca confidenciando seus problemas a outros, maior estresse e não morar sozinho. Kibret et al. (2020) obtiveram resultados semelhantes, dos 305 profissionais de saúde na Etiópia estudados, ser casado foi estatisticamente associado à ansiedade.

Para tal resultado, Tsaras et al. (2018), obtiveram resultados divergentes, em que observaram que enfermeiras solteiras eram 4. 63 vezes mais propensas de desenvolver um risco para transtorno de ansiedade em comparação com enfermeiras casadas.

A respeito da experiência profissional, conforme Sahin et al. (2020), em um estudo realizado com 939 profissionais de saúde na Turquia, os escores de depressão e ansiedade em participantes que trabalharam por 10 anos ou mais foram significativamente maiores do que aqueles que trabalharam por menos de 10 anos. Os enfermeiros apresentaram escores de insônia e angústia mais altos do que os outros profissionais.

Também foram observados a presença de transtornos de ansiedade aos fatores associados a pandemia de COVI-19. Durante a epidemia, o trabalhador teve alta prevalência de sintomas de ansiedade autorreferidos (Liu et al., 2021). Conforme Azoulay et al. (2020) e Zhou et al. (2020), os prestadores de serviços de saúde da linha de frente que gerenciam pacientes com doença coronavírus exibem uma alta prevalência de sintomas de transtornos de saúde mental em comparação com a população em geral, apresentando maior probabilidade de desenvolver depressão, ansiedade, somatização e insônia.

No estudo de Wang et al. (2020), os autores mostram que profissionais de saúde da linha de frente; que tinham treinamento e recursos insuficientes para proteção; falta de confiança nas medidas de proteção; preocupadas com a infecção; e 
Enfermeiros, foram estatisticamente associados a ansiedade. O medo de ser infectado também foi observado por Azoulay et al. (2020).

Corroborando com o estudo de Sahin et al. (2020), mostram que todas as pontuações foram maiores nos trabalhadores da linha de frente da Covid-19. Foi observado também pontuações mais altas entre os participantes que foram testados para Covid-19, em comparação com aqueles que não o fizeram o teste. Os escores de depressão e angústia foram significativamente maiores entre os profissionais que não contraíram a doença COVID-19.

Foi observado uma relação dos sintomas ansiosos e a preocupação com a família, como o estudo de Kibret et al. (2020) e Amin et al. (2020), a respeito disso, Hacimusalar et al. (2020) obtiveram resultados semelhantes, em que no estudo realizado com 2.156 participantes na Turquia, dos participantes, 52,0\% (n: 1121) eram profissionais de saúde, os níveis de ansiedade e desesperança foram maiores nos que viviam com um indivíduo de alto risco em casa durante a pandemia, nas que tinham dificuldade em cuidar dos filhos, e aqueles cuja renda diminuiu. Azoulay et al. (2020), realizado com profissionais de UTI na França obtiveram resultados semelhantes, em que os sintomas de ansiedade aumentaram significativamente nos profissionais que não tinham tempo de cuidar de sua própria família.

Por fim, com relação a jornada de trabalho observado no estudo de Amin et al. (2020), Zhou et al. (2020) encontraram resultados semelhantes, um total de 606 funcionários de hospitais de 133 cidade da China, em que as horas de trabalho elevadas diárias foram associadas a sintomas de ansiedade na equipe médica da linha de frente. No estudo de Azoulay et al. (2020) observaram que os sintomas de ansiedade foram elevados em profissionais que não tinham tempo para descansar em decorrência da jornada de trabalho. Conforme Hacimusalar et al. (2020), o aumento da jornada de trabalho é um dos fatores importantes que afetam a ansiedade.

\section{Prevalência dos principais transtornos ansiosos em comparação e/ou associação a outros transtornos mentais}

Os transtornos ansiosos vêm afetando diversas pessoas em todo o mundo, neste aspecto, os profissionais da saúde são suscetíveis ao seu aparecimento e a outros transtornos mentais, onde o fato se deve a associação de diversos fatores de risco como: idade, sexo, estado civil e presença de doenças crônicas e outros (Kibret et al., 2020).

Corroborando com a afirmativa, Zhou et al. (2020) sustenta a ideia de que os profissionais da saúde são afetados principalmente por ansiedade, depressão, somatização e insônia, ou ainda, potencial risco para cometer suicídio. O que se caracteriza como um grave problema que pode afetar profissionais de todo o mundo, onde deve-se diminuir os fatores de risco e destacar os fatores de proteção.

Evidenciando que os profissionais da saúde precisam receber apoio social e utilizar estratégias para minimizar os riscos de aparecimento de transtornos mentais. Hennein et al. (2021) observaram em sua pesquisa que, os profissionais apresentaram sinais e sintomas de provável ansiedade generalizada, depressão, provável transtorno de estresse pós-traumático e propensão a transtornos de abuso de álcool. Comprovando a necessidade de cuidar da saúde mental dos profissionais.

Segundo Campo et al. (2016) a ansiedade e depressão podem ocasionar outros agravos a saúde, como distúrbios musculoesqueléticos, onde Tsaras et al. (2018), afirma que a ansiedade e depressão são transtornos mentais mais prevalentes em trabalhadores da saúde, no qual muitas vezes, podem estar associados ao ambiente de trabalho e fatores socioeconômicos. Corroborando com as afirmativas, Amin et al. (2020) em sua pesquisa com profissionais da saúde no período da pandemia, constatou que a prevalência de ansiedade e depressão chegou a $43 \%$ dos participantes do estudo. E Azoulay et al. (2020) acrescentam que a ansiedade, depressão e transtorno pós-traumático se relacionaram com altas cargas de trabalho, incapacidade de descansar durante o horário de trabalho, insegurança, insônia e medo.

Onde a desesperança também foi ligada a diversos transtornos mentais, principalmente a ansiedade, pois foi observado que a mesma é um dos primeiros sintomas para o aparecimento de outros transtornos, colaborando para o declínio 
da saúde mental dos trabalhadores juntamente a fatores de risco como: a preocupação com a família, baixos salários, trabalho com pacientes de covid-19 e aumento das cargas de trabalho (Hacimusalar et al., 2020). No qual, Satin et al. (2020), destaca ainda a insônia, angústia, ansiedade e depressão como principais agravos a saúde mental.

\section{Estratégias de prevenção/proteção para transtornos ansiosos em profissionais da saúde}

Entre as estratégias que podem prevenir ou proteger os profissionais da saúde dos transtornos ansiosos, a harmonia, apoio e colaboração entre a equipe é fundamental para manter a resiliência, assim como ter meios para manter um apoio social estável (Hennein et al., 2021).

De acordo com Wang et al. (2020), um treinamento eficaz e o fornecimento de EPI's adequados torna os profissionais menos inseguros e menos ansiosos. Corroborando com esta afirmação, Amin et al. (2020) relatam que os EPI's e práticas de controle de infecção são ideais, além disso, os gestores devem garantir cobertura médica tanto para os profissionais como para suas famílias; intervenções psicológicas, como terapia cognitiva-comportamental, e avaliar se tais infecções tão tendo eficácia sobre o bem-estar psicológico dos trabalhadores.

No cenário da pandemia, é importante que as instituições façam uso da telepsicologia, garantindo atendimento virtual para os profissionais da linha de frente ou introduzam medidas de apoio psiquiátrico dentro dos próprios hospitais. É importante também que se tenha um grupo de suporte virtual, com profissionais de saúde mental que ofereçam escuta ativa, resolutividade de problemas, práticas de higiene do sono, entre outros (Hennein et al., 2021; Sahin et al., 2020)

\section{Considerações Finais}

Este estudo permitiu observar a predisposição dos transtornos ansiosos em profissionais de saúde, através de uma revisão de literatura, na qual foi avaliada a prevalência dos principais transtornos ansiosos em comparação e/ou associação a outros transtornos mentais, destacando estratégias de prevenção para transtornos ansiosos e relacionando o surgimento da pandemia de Covid-19 com o agravamento da saúde mental de profissionais da saúde.

Diante dos problemas que foram encontrados, torna-se claro que os profissionais da saúde também precisam receber assistência física e psicológica, além de apoio social e implementação de estratégias que visem minimizar o risco de aparecimento de transtornos mentais.

Neste aspecto, deve-se destacar ainda que, frequentemente a ansiedade vem relacionada a outros transtornos mentais como: depressão, e estresse pós-traumáticos. Transtornos estes que estão ligados diretamente com diversos fatores de risco como: altas cargas de trabalho, equipamentos de proteção individual em quantidade insuficiente, preocupação com a família, idade, experiência profissional, doenças crônicas e outros. Como limitações do estudo tem-se o fato de que muitas vezes, os profissionais não recebem diagnósticos ou ainda, por se tratar de um tema delicado, não aceitam participar de pesquisas.

Por fim, o estudo possibilitou expandir os conhecimentos acerca dos transtornos ansiosos e outros transtornos mentais que poder acometer os profissionais de saúde no exercício do trabalho, destacando ainda, o aumento do número de casos decorrentes da pandemia. Dessa forma, considera-se que mais pesquisas sejam feitas nesta linha de investigação, a fim de comprovar a importância do acompanhamento psicológico a profissionais de saúde, evidenciando a necessidade da utilização de técnicas para proteger a saúde mental dessa classe profissional tão essencial a toda a sociedade.

\section{Referências}

Alves, D. L., \& Oliveira, F. B. M. (2016). Relação entre a sobrecarga de trabalho e erros de administração de medicação na assistência hospitalar. Revista Ciências \& Saberes, 2(2).

Amin, F., Sharif, S., Saeed, R., Durrani, N., \& Jilani, D. (2020). COVID-19 pandemic- knowledge, perception, anxiety and depression among frontline doctors of Pakistan. BMC Psychiatry, 20(459), 1-9. 
Azoulay, E., Cariou, A., Bruneel, F., Demoule, A., Kouatchet, A., Reuter, D., \& Kentish-Barnes, N. (2020). Symptoms of Anxiety, Depression, and Peritraumatic Dissociation in Critical Care Clinicians Managing Patients with COVID-19. A Cross-Sectional Study. American journal of respiratory and critical care medicine, 202(10), 1388-1398.

Bork, A. M. T. (2011). Enfermagem baseada em evidências. Rio de Janeiro: Guanabara Koogan.

Del Campo, M. T., Romo, P. E., Hoz, R. E. D. L., Villamor, J. M., \& Mahíllo-Fernández, I. (2017). Anxiety and depression predict musculoskeletal disorders in healthcare workers. Arch Environ Occup Health, 72(1), 39-44.

Hacimusalar, Y., Kahve, A. C., Yasar, A. B., \& Aidin, M. S. (2020). Anxiety and hopelessness levels in COVID-19 pandemic: A comparative study of healthcare professionals and other community sample in Turkey. J Psychiatr Res., 129, 181-188.

Hennein, R.; Mew, E. J., \& Lowe, S. R. (2021). Socio-ecological predictors of mental health outcomes among healthcare workers during the COVID-19 pandemic in the United States. PLOS ONE, 16(2), 1-18.

Kibret, S., Teshome, D., Fenta, E., Hunie, M., \& Tamire, T. (2020). Prevalence of anxiety towards COVID-19 and its associated factors among healthcare workers in a Hospital of Ethiopia. Plos One, 15(12).

Liu, Y., Chen, H., Zhang, N., Wang, X., Fan, Q., Zhang, Y., \& Li, M. (2021). Anxiety and depression symptoms of medical staff under COVID-19 epidemic in China. Journal of Affective Disorders, 278, 144-148.

Machado, M. B., \& Quevedo, J. (2016). Prevalência de transtornos ansiosos e algumas comorbidades em idosos: um estudo de base populacional. J. bras. psiquiatr., 65(1), 28-35.

Melo, K. C., Da Silva, C. O., Souza, M. W. S. S., Soares, A. N., Da Silva, W. C., Oliveira, F. B. M., \& Da Silva, R. A. (2021). Síndrome de burnout em profissionais da saúde que atuam na linha de frente da covid-19. International Journal of Development Research, 11(04), 44706-44711.

Moura, A., Lunard, R., Volpato, R. J., \& Nascimento, V. F. D. (2018). Fatores associados à ansiedade entre profissionais da atenção básica. Revista Portuguesa de Enfermagem de Saúde Mental, (19), 17-26.

Park, C., Hwang, J. M., Jo, S., Bae, S. J., \& Sakong, J. (2020). COVID-19 Outbreak and Its Association with Healthcare Workers' Emotional Stress: a CrossSectional Study. J Korean Med Sci., 35(41), 1-10.

Sahin, M. K., Aker, S., Sahin, G., \& Karabekiroğlu, A. (2020).Prevalence of depression, anxiety, distress and insomnia and related factors in healthcare workers during covid-19 pandemic in turkey. Journal of community health, 45(6), 1168-1177.

Santana, C. C., \& Bião, M. A. S. (2018). Eficácia do neurofeedback no tratamento da ansiedade patológica e transtornos ansiosos: revisão sistemática da literatura. Psic., Saúde \& Doenças, 19(2), 234-242.

Da Silva, W. C., Da Silva, C. O., Melo, K. C., Soares, A. N., Hernandes, L. F., Araújo, Z. A. M., \& Sousa, F. D. C. A. (2021). Explorando os impactos na saúde mental de crianças durante a pandemia de covid-19. International Journal of Development Research, 11(04), 46248-46253.

Sousa, L. M. M., Vieira, C. M., Severino, S., \& Antunes, V. (2017). A metodologia de revisão integrativa da literatura em enfermagem. Revista de Investigação de Enfermagem, 7-26.

Tsaras, K., Papathanasiou, I. V., Vus, V., Panagiotopoulou, A., Katsou, M. A., Kelesi, M., \& Fradelos, E. C. (2018). Predicting Factors of Depression and Anxiety in Mental Health Nurses: A Quantitative Cross-Sectional Study. Med Arch, 72(1), 62-67.

Wang, Y., Ma, S., Yang, C., \&Cai, Z. (2020). Acute psychological effects of Coronavirus Disease 2019 outbreak among healthcare workers in China: a crosssectional study. Translational Psychiatry, 10(348), 1-10.

Zhou, Y., Wang, W., Sun, Y., Quian, W., Liu, Z., Wang, R., \& Zhang, X. (2020). The prevalence and risk factors of psychological disturbances of frontline medical staff in china under the COVID-19 epidemic: Workload should be concerned. Journal of affective disorders, 277, 510-514. 